\title{
Les appellations du nationalisme politique écossais au vingtième siècle
}

Labelling Scottish Political Nationalism in the Twentieth Century

\section{Arnaud Fiasson}

\section{(2) OpenEdition}

12 Journals

Édition électronique

URL : http://journals.openedition.org/etudesecossaises/1013

DOI : 10.4000/etudesecossaises.1013

ISSN : 1969-6337

Éditeur

UGA Éditions/Université Grenoble Alpes

\section{Édition imprimée}

Date de publication : 25 avril 2015

Pagination : 153-172

ISBN : 978-2-84310-296-7

ISSN : 1240-1439

Référence électronique

Arnaud Fiasson, "Les appellations du nationalisme politique écossais au vingtième siècle », Études écossaises [En ligne], 17 | 2015, mis en ligne le 25 avril 2016, consulté le 16 mars 2021. URL : http:// journals.openedition.org/etudesecossaises/1013; DOI : https://doi.org/10.4000/etudesecossaises. 1013 


\section{Les appellations du nationalisme politique écossais au vingtième siècle}

La prolifération d'organismes nationalistes en Écosse coïncide avec le phénomène linguistique d'abrègement apparu dans les années 1920 et 1930. Devenus manifestes en particulier au sein de la langue française, les sigles et les acronymes ont évolué d'un état de groupes d'initiales abréviatives au statut de procédés stylistiques plus ou moins usités (Himelfarb, 2002, p. 6). À la différence que l'acronyme constitue une forme lexicalisée et oralisée du sigle, ces deux types d'abréviation peuvent faire l'objet de jeux de mots et ainsi induire un changement de sens, ou risquent parfois de remplacer la dénomination propre de l'objet (Dupriez, 1984, p. 15-17, 23). Sources de confusion, ils sont ainsi susceptibles de déformer le sens convoqué par une appellation particulière. Un exemple marquant, que l'on trouve aussi bien en langue française qu'anglaise, est celui de l'utilisation de «Parti nationaliste écossais» pour expliciter le sigle SNP qui signifie pourtant «Scottish National Party». De la même manière, l'extrait suivant tiré du roman And the Land Lay Still écrit par James Robertson présente la faculté de synthèse d'un protagoniste qui réduit les faits historico-politiques à leur forme la plus simple, comme en témoigne sa conception de l'échiquier politique nationaliste écossais dans les années 1970 :

Basically, take a handful of letters, including an S, an R and an L, maybe a $\mathrm{W}$ if you really need to emphasise your left-wing credentials, fling them up in the air and when they come down you've got a new movement. Same individuals, different organisation. [...] They have a shotgun and a couple of air rifles, if they're lucky an ex-REME guy who knows how to wire up a bomb. But do these people have anything to do with the Scottish National Party? No, they despise the SNP. (Robertson, 2010, p. 304)

Dans la mesure où «l'appellation révèle, montre, caractérise, en même temps qu'elle exclut, gomme et préjuge ${ }^{1}{ }^{\prime}$, la caractérisation du mouve-

1. Texte de cadrage du 53 Congrès de la SAES «Appellation(s)», 17-19 mai 2013, université de Bourgogne, Dijon. 
ment nationaliste écossais peut parfois se révéler problématique si l'on s'en tient au point de vue adopté par ses acteurs. Par exemple, lorsque John MacCormick écrit à Duncun McMeill (nom de plume de Neil Gunn) pour lui faire part des faibles résultats électoraux du National Party of Scotland, il ajoute :

As you know my own outlook in politics is radical, and I know I cannot help letting that Radicalism show through my Nationalist speeches. [...] I know the feelings of our members pretty well, or those who are worth anything to us, former Conservatives as well as former Socialists, and I believe that they are all Radicals [...]. Is it possible that a National Radical Party might make a more immediate and wider appeal to the electors than a National Party $[\ldots]$ ?

Dans une lettre également adressée à Neil Gunn, Tom Gibson s'inscrit en faux contre ce radicalisme autoproclamé lorsqu'il exprime une opinion défavorable au sujet de la stratégie conciliatoire de MacCormick, qui vise à réduire les tensions idéologiques au sein du parti.

MacCormick has given people the impression that he is weak on this point and I do feel a more definite line on his part towards this important matter will go a long way to relieve the present situation. [...] I would seriously suggest to you that you influence MacCormick on this point.

MacCormick propose donc une vision du radicalisme qui convient à son propre programme politique et qui est justifiée par des circonstances partisanes, bien qu'elle ne soit pas partagée par tous. Cet exemple montre que les appellations attribuées aux organisations nationalistes dans certains documents de source primaire sont susceptibles d'apparaitre comme autant de points de discorde, notamment en raison de la subjectivité propre aux acteurs de la scène politique nationaliste qui ne disposent pas de recul historique sur les événements qui leur sont contemporains.

Loin de dresser une liste exhaustive des différentes manifestations politiques relatives au nationalisme écossais au vingtième siècle, cet article s'interroge, à la lumière d'une distanciation historique et critique, sur les appellations qui leur conviendraient le mieux. Il s'agit de proposer une classification objective fondée non seulement sur des définitions linguistiques attestées mais également sur la base de faits objectifs amenés par l'étude des idéologies et des répertoires d'action dont usent les individus et les organisations se revendiquant du nationalisme politique écossais. En raison de leur récurrence dans le discours nationaliste et dans le but de fournir un cadre conceptuel, les appellations «extrémiste», «radical» et «terroriste» ont été retenues en vue de prolonger l’interprétation de la 
notion de nationalisme politique avancée par Robert Thomsen². L'organisation chronologique qui est privilégiée tente quant à elle de rendre compte de l'évolution du nationalisme politique au cours du vingtième siècle. De ce fait, cet article propose d'appliquer une classification onomastique aux quatre phases principales que nous discernons dans l'histoire du mouvement nationaliste écossais. Notre approche, essentiellement historique, est complétée d'une exemplification qui s'appuie sur des extraits tirés de correspondances privées, de la littérature nationaliste, de communiqués, de rapports officiels et d'articles de presse.

\section{Création et positionnement du nationalisme politique écossais}

La cristallisation du sentiment national écossais en une manifestation politique du nationalisme moderne s'opère au cours de la seconde moitié du dix-neuvième siècle et par la formation de la National Association for the Vindication of Scottish Rights (NAVSR). Gette association, créée en 1853 suite à la famine consécutive à la maladie de la pomme de terre, marque la première apparition en Écosse d'une organisation politique en faveur d'un changement constitutionnel. Bien que la NAVSR reçoive le soutien de quelques députés libéraux et conservateurs, elle reste éphémère et peu influente sur la scène politique britannique (Leruez, 2000, p. 154). L'intérêt autonomiste est ensuite ravivé en 1885 par le retour du poste de secrétaire d'État à l'Écosse (disparu après la révolte jacobite de 1745) et par la création du Scottish Office.

Le nationalisme politique s'affirme dès le début du vingtième siècle par la création de la Young Scots Society ${ }^{3}$, affiliée au parti libéral. L’enthousiasme ${ }^{4}$ manifeste de cette société, amplifié par l'élection à Westminster

2. R. G. Thomsen, Nationalism in Stateless Nations, Birlinn Ltd, 2010, p. $8:$ : [P]olitical nationalism includes the political expression of demands for constitutional change; it also refers to the desire for the concession of power in the shape of devolution, home rule, or independence. It is impossible to say precisely when a given variant of nationalism becomes political, but for it to be so, it will obviously have to address questions and situations politically, either within the established political party system or in other political forums. »

3. Le sigle YSS n'apparaît pas dans les documents publiés par la Young Scots Society, mais peut figurer dans des ouvrages universitaires. Voir, par example, J. Kennedy, Liberal Nationalisms: Empire, State, and Civil Society in Scotland and Quebec, McGuill-Queen's University Press, 2013, p. 57-63.

4. W. Laughland, Manifesto and Appeal to the Scottish People on Scottish Home Rule, Young Scots Society, 1911, p. 4 : «There must be steady, persistent effort, and no weak-kneed policy can be allowed to endanger the cause. Earnest men, loyal patriots are required at this moment of all moments in the history of the nation, and if necessary the Young Scots Society is ready to fight, and bring about the defeat if anyone who, through fear or half-heartedness, might imperil Scotland's chances of regaining the precious privilege and right of making and administering her own laws. » 
d'un total de trente de ses candidats sur la période entre 1905 et 1914, fait qu'ils peuvent présenter un certain nombre de projets de loi en faveur d'une autonomie gouvernementale limitée ${ }^{5}$. L'un des projets de loi est voté en seconde lecture en mai 1914 mais s'enlise à l'approche de la Première Guerre mondiale (Devine, 2006, p. 306-308).

En 1918, dès la reprise de la Scottish Home Rule Association (SHRA) sous la tutelle financière de Roland Eugene Muirhead ${ }^{6}$ et ancien membre de la Young Scots Society, le nationalisme écossais prend un nouvel élan. L'affiliation de Muirhead, ainsi que celle d'un certain nombre de membres de l'association, à l'Independent Labour Party (ILP) place rapidement la SHRA à gauche dans l'échiquier politique. Le nationalisme politique, par l'intermédiaire de ce groupe de pression parlementaire, rend compte de l'évolution du sentiment national en force politique à partir de l'après-guerre, comme en témoigne la résolution suivante votée en 1918.

That this meeting, representing all shades of Scottish opinion and industrial activity, being convinced that the present centralised system of government from London is inefficient and inconsistent with national sentiment, resolves to form itself into a committee for the purpose of organising and focusing the Scottish demand for self-government in respect of Scottish affairs. (Civardi, 1998, p. 198)

On remarque que le sentiment national est étroitement lié au mécontentement à l'égard du système politique britannique, établissant ainsi les bases sur lesquelles s'appuie la demande d'autonomie. En d'autres termes, la SHRA n'invoque pas seulement les différences culturelles à l'origine du nationalisme écossais mais permet la création d'une voix politique de celui-ci par le désir d'agir au sein de l'institution parlementaire britannique, comme en témoigne un extrait tiré de la correspondance privée de Muirhead : "The idea of the Executive is to make this a genuine all-Scotland non-party Association for the single purpose of getting Home Rule at the earliest possible date. ${ }^{7} \gg$ Cette voix politique se concrétise en 1924 par la création de la Scottish National Convention (SNG) qui est en charge de rédiger plusieurs projets de loi à visée autonomiste et dont le plus notable est celui présenté à la Chambre des communes par le député travailliste George Buchanan. Toutefois, chaque projet est rejeté en raison du manque de soutien des députés écossais, ce qui laisse

5. Ibid., p. 1. Les objectifs de la société sont les suivants : «to further the National interests of Scotland, and to secure for Scotland the right of self-government».

6. Voir NLS, Acc 3721, Papers of the Scottish Secretariat and of Roland Eugene Muirhead. Il signe ses lettres par le sigle REM.

7. NLS, Acc 3721/78/2, Lettre de R. E. Muirhead à J. M. Hogge, 27 février 1919. 
présager le caractère vain d'un groupe de pression parlementaire dans le contexte des années 1920.

Alors que la Young Scots Society et la SHRA affichent une forme de conventionnalisme institutionnel en raison de leur répertoire d'action qui se consigne au champ législatif, ces organisations se rapportent au radicalisme politique puisqu'elles «mettent en question les dispositions existantes, demandent des réformes, ou l'abolition de ce qui ne leur semble pas justifié sur le plan des principes» (Coleman et al., 1989, p. 637). À l'instar de William Gladstone qui proposa la mise en place d'un parlement autonome en Irlande en 1885, la YSS et la SHRA appellent à la réforme du système politique britannique dans son ensemble et soulignent que l'autonomie irlandaise doit s'accompagner de l'autonomie des autres parties constituantes du Royaume-Uni. Leur projet réformiste, qu'ils justifient par un idéal démocratique ${ }^{8}$, demande la création d'un parlement écossais autonome qui serait plus à même de gérer les affaires écossaises qu'un parlement britannique déjà surchargé par la gestion de l'Empire (Mitchell, 2014, p. 88-92).

Par opposition à la SHRA, la Scots National League (SNL) adopte une idéologie et une stratégie différente dès sa création en 1920 puisqu'elle se place clairement en faveur de l'indépendance écossaise. D’abord préoccupée par l'enseignement de l'histoire écossaise, par la propagation du sentiment que l'Écosse n'est autre qu'une colonie de l'Angleterre et par le statut de la langue gaélique ${ }^{9}$, elle change radicalement d'orientation dès l'arrivée de Tom Gibson à partir de 1924 (Finlay, 1994, p. 38-47). En effet, les objectifs de la SNL se retrouvent dans sa constitution :

$[\ldots]$ the resumption of Scottish National Independence [...] that Scotsmen acting nationally have the sole right to formulate and finally decide upon what scheme of self-government for Scotland should be adopted. (Brand, 1978, p. 185)

Tandis que cela ne suffit pas à définir la SNL en tant que parti politique, il faut remarquer la finalité indépendantiste de l'organisation et

8. NLS, Acc 3721/79, Minutes of the SHRA, fifth annual meeting, 21 avril $1923:$ «[T] he time is ripe for a reconsideration by the people of Scotland of the conditions under which they stand bound together with the other parts of the United Kingdom. [...] [T] he Scottish people, being a Nation in the full sense of the term, ought to have a means of organising, formulating, and giving effect to its national will, and [...] a Scottish Parliament democratically elected, and sitting in Scotland, is essential for this.»

9. Durant sa première période (1920-1924), la SNL était «an organisation comprised of Gaelic romantics, concerned with the teaching of Scottish History, Scotland's perceived position as a colony of England and the status of the Gaelic language» (Lynch, 2002, p. 33). Cela montre que la SNL fut premièrement l'expression de la dimension culturelle du nationalisme avant l'arrivée de Tom Gibson en 1924. 
l'importance qui est accordée au pouvoir de la nation. Par ailleurs, en 1926, la SNL lance le journal The Scots Independent (SI) et l'utilise comme organe de campagne et de communication ${ }^{10}$. D'un autre point de vue, l'instauration de l'exclusivité d'adhésion au sein de la SNL empêche tout membre d'être affilié à un parti politique et représente en outre les prémisses de l'établissement d'un parti (Lynch, 2002, p. 34). La SNL ne se veut pas être un parti en tant que tel, au grand dam de son dirigeant Tom Gibson qui propose de modifier l'appellation ${ }^{11}$ de la ligue par Scots National Party et qui, non content de ne pas recevoir le soutien des membres, décide tout de même de publier un livret de propagande au nom du Scots National Party, avant de se faire reprendre par le comité exécutif de la Ligue deux mois plus tard. L'entêtement de Tom Gibson est motivé par la motion qu'il propose et qui est adoptée lors de la conférence annuelle de la Ligue le 15 juin 1925 :

[...] the policy of the Scots National League is, by means of the existing electoral activity, to obtain a majority of Scottish representatives pledged to remain on Scottish soil and to resume the powers of government in Scotland and that national candidates independent of all political parties be put forward at the next General Election ${ }^{12}$.

On note ici la teneur radicale de la SNL qui s'aligne sur le mouvement nationaliste irlandais, dans le sens qu'une fois qu'une majorité de parlementaires écossais serait atteinte, cela représenterait pour la SNL le mandat qui rendrait légitime le retour de ces députés en Écosse, quittant ainsi Westminster, dans le but de rouvrir le parlement d'Écosse.

Le Scottish National Movement (SNM), groupe dissident de la SNL en raison de l'orientation politique adoptée par cette dernière, fut créé le

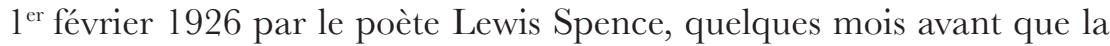
déclaration de Balfour confère aux dominions et à la Grande-Bretagne le statut de «communautés autonomes au sein de l'Empire britannique ${ }^{13}$ »

10. NLS, Acc 6058/2/1, Minutes of the National Executive, 14 août 1926 :

«1. Ownership: The paper shall be the official organ, and the exclusive property of the League. 2. Object: The sole object of publication shall be the furtherance of the aim and policy of the League. »

11. NLS, Acc 6058/2/1, Minutes of the Annual Conference, 12 juin 1926 : «Mr Gibson on behalf of the Glasgow Branch withdrew the resolution as to change of nomenclature, but reserved their right to propose such a change at a future date.»

12. NLS, Acc 6058/2/1, Minutes of the Annual Conference, 10 juin 1925.

13. Inter-Imperial Committee, Report, Proceeding, Memoranda, Imperial Conference, 1926, p. 3, (<http://foundingdocs.gov.au/resources/transcripts/cth11_doc_1926.pdf>) : «[T] he group of selfgoverning communities composed of Great Britain and the Dominions [...] are autonomous Communities within the British Empire, equal in status, in no way subordinate one to another in any aspect of their domestic or external affairs, though united by a common allegiance to the Crown, and freely associated as members of the British Commonwealth of Nation. » 
- signal annonciateur de la transformation progressive de l'Empire en Commonwealth. Le SNM entend obtenir l'indépendance par le «rétablissement du parlement écossais», mais sans passer par Westminster : il appelle à la mobilisation de toutes les composantes de la société écossaise à exprimer leur revendication indépendantiste par le biais d'un plébiscite et de s'adresser au roi George V (Spence, 1927, p. 40). Hormis la revendication de l'indépendance par le rétablissement du parlement, les objectifs de la SNM, détaillés dans le pamphlet Freedom for Scotland, sont triples : éduquer, par la dissémination de propagande, les Écossais sur les questions de première importance telle que la notion de terre et de langue en vue de démontrer que l'Écosse s'est laissée coloniser par l'Angleterre; raviver le sentiment national par l'enseignement de l'histoire écossaise; promouvoir l'art, la littérature et la musique écossaise. S'il l'on en juge par la préface, le tout repose sur un sentiment anti-Anglais (Spence, 1927, p. 1, 31, 38, 41). Si nous retenons la définition selon laquelle l'extrémisme correspond à «['a]ttitude politique dénonçant le fonctionnement de la démocratie représentative (règles de compétition, modes de représentation, mécanismes de décision) et prônant le recours à des formes d'action politique directes et radicales, et parfois violentes» (Nay, 2008, p. 210), le SNM se détache du radicalisme avancé par les organisations mentionnées plus haut. Plus particulièrement, Spence tient le discours suivant devant le comité de la SNC en 1927 :

Now what we have to propose is the following, which I will put before you in a very few words: - We do not yet advocate taking up the rifle, because that is a course I feel that would hardly appeal to the majority of our fellow countrymen - although it is the method that I would personally prefer. I will not deny that for a moment. I believe the only way to beat an Englishman is to beat him physically. You cannot drive it into the thick head of the Southerner by any other means ${ }^{14}$.

Spence confirme ainsi les lignes extrémistes suivies par le SNM en déclarant, à titre individuel, son parti pris pour l'utilisation de la violence armée, et ce au détriment de l'opinion majoritaire des Écossais. De la même manière que le SNM se concentre sur la dimension culturelle du nationalisme, mais sans toutefois tendre à l'extrémisme politique, la Glasgow University Student Nationalist Association (GUSNA), fondée en 1927 par John MacCormick, établit ses objectifs comme étant «l'autodétermination de l'Écosse» et «l'avancement des idéaux de la culture écossaise à l'intérieur comme à l'extérieur de l'université» (Brand, 1978, p. 192). Il

14. NLS, Acc 3721/81, Report of the Meeting of the Committee of Convention Held in the Christian Institute, Glasgow, 29 mai 1927, p. 13. 
faut remarquer le caractère ambigu du terme autodétermination («selfgovernment») qui inclut à la fois une forme d'autonomie gouvernementale plus ou moins limitée («Home Rule») et d’indépendance (totale ou au sein de l'Empire britannique ou du Commonwealth à venir). Par opposition, la promotion du caractère culturel du nationalisme est univoque, sans toutefois verser dans les considérations polémiques avancées par Spence.

\section{Polarisation du nationalisme politique écossais}

La cause nationaliste se polarise lors de la création du National Party of Scotland (NPS) en 1928. Le NPS, dont la composition du bureau ${ }^{15}$ témoigne de l'étendue des compromis réalisés entre la SHRA, la SNL, le SNM et la GUSNA, constitue un parti au sens propre du terme, c'est-à-dire une organisation politique visant à soumettre des candidats aux élections législatives, en vue de les faire élire à la Chambre des communes ${ }^{\mathbf{1 6}}$. Le NPS, qui devient l'unique organisation politique nationaliste en Écosse, subit toutefois l'épreuve d'une polarisation interne qui oppose progressivement les radicaux aux modérés en raison d'un objectif vaguement défini comme étant «self-government for Scotland with independent national status within the British group of nations » que les membres interprètent comme autonomie plus ou moins limitée ou indépendance, au sein du Commonwealth ou non. Selon Arthur Donaldson, la division stratégique est également complétée par une division idéologique ${ }^{17}$ supplémentaire amenée par l'extrémisme de Christopher Murray Grieve qui promeut fascisme et violence (Finlay, 1994, p. 80-85).

La polarisation du nationalisme se renforce et dépasse le confinement au NPS dès l'émergence du Scottish Party (SP) placé sous la coupe du duc de Montrose. Créé en 1932, après le vote du Statute of Westminster en décembre 1931, le SP, dont les membres sont affiliés au parti conservateur et au parti libéral, s'inscrit dans une dynamique d'autonomie gouvernementale au sein de l'Empire britannique. Il s'oppose ainsi à l'orientation indépendantiste ${ }^{18}$ qui a progressivement gagné un NPS situé à gauche

15. Le bureau est composé des têtes de file des organisations nationalistes précédentes: Grahame, Muirhead, Spence, MacCormick, Valentine et Gibson.

16. Le NPS n'est pas exempt de tensions entre les anciens membres de la SHRA d'une part, en faveur de l'établissement d'un groupe de pression parlementaire en vue de l'obtention de l'autonomie, et les anciens membres de la SNL d'autre part, qui favorisent une indépendance totale par l'intermédiaire d'une stratégie de compétitivité électorale.

17. NLS, Acc 3721/5/76, Lettre de Roland E. Muirhead à Arthur W. Donaldson, 16 juin 1930.

18. NLS, Acc 3721/94/67, Lettre des membres du bureau du NPS à Roland E. Muirhead, 6 juin 1933 : «We must therefore call upon the party, to purge itself of an undesirable element, with whom 
dans l'échiquier politique en raison de ses affiliations au parti travailliste et au parti travailliste indépendant. La correspondance privée entre Donaldson et Muirhead témoigne de la polarisation grandissante de la scène politique nationaliste.

The place for the Moderates and Imperialists is with the Duke of Montrose and his crew. [...] Personally, I am for Scottish Nationalism without qualification or impediment of any kind, and my support will always be for the element which seeks independence ${ }^{19}$.

Tandis que Donaldson met un point d'honneur à se distancier de la modération politique, Muirhead remarque qu'une fusion avec le Scottish Party semble remporter un soutien considérable au sein du NPS et que cette fusion apparaît préférable en vue de réunir le nationalisme écossais $^{20}$. Si Gibson est peu enthousiaste à cette démarche, il affiche toutefois des propos extrémistes lorsqu'il n'exclut pas une forme d'assimilation des membres du SP :

[...] they [SP members] have only a nuisance value. Before the Movement can progress they must be got rid of either by smashing them or by absorption. It is perfectly true that we may transform them or some of them from a definite liability to a more or less asset, but even if some of them may remain liabilities we can best deal with them from the inside. To allow them to remain as at present would be fatal ${ }^{21}$.

Tandis que Gibson ne prône pas nécessairement la violence, il reste néanmoins convaincu que la fin justifie les moyens pour empêcher l'ascendant du SP, favorisé par l'agenda conciliatoire de MacCormick, comme nous l'avons remarqué dans l'introduction à cet article. C'est pourtant la combinaison des spécificités de chaque parti qui servira de moteur à la création du SNP en 1934 puisque la fusion procure au NPS l'expérience politique qui lui faisait défaut, en plus de contenter les nombreux autonomistes dans les rangs du parti. D'autre part, le SP ne comptant que très peu de membres, la fusion est perçue comme le moyen d'acquérir un réel impact électoral. Dans la mesure où les deux partis s'opposent sur leurs

continued association is impossible as otherwise our own position within the Party will become intolerable.» La purge se concrétise par l'expulsion de deux membres qui critiquent le dévolutionisme et le «pur carriérisme» dont font soi-disant preuve les membres du bureau.

19. NLS, Acc 3721/5/76, Lettre d'Arthur W. Donaldson à Roland E. Muirhead, 20 février 1933.

20. NLS, Acc 3721/5/76, Lettre de Roland E. Muirhead à Arthur W. Donaldson, 8 mars 1933 : «Before the moderate Party came into existence, practically all those who were in favour of a National Parliament for Scotland had joined the National Party, although a proportion of our members would have preferred something like a Devolution scheme those members are now suggesting an amalgamation with the Scottish Party.»

21. NLS, Acc 3721/93/55, Lettre de Tom H. Gibson à Roland E. Muirhead, 14 janvier 1934. 
bases idéologiques, les compromis et les discussions seront à nouveau de rigueur.

\section{Radicalisation et modération du nationalisme politique écossais}

Nous retrouvons l'étendue des compromis entre les deux forces politiques nationalistes dans la constitution du SNP :

(1) The establishment of a Scottish Parliament which shall be the final authority on all Scottish affairs, including taxation.

(2) Scotland shall share with England the rights and responsibilities they, as mother nations, have jointly created and incurred within the British Empire.

(3) In a manner representing the will of her people, Scotland should set up jointly with England, machinery to deal with these responsibilities and in particular with such matters as Defence, Foreign Policy and the creation of a customs union.

(4) It is believed that these principles can be realised only by an independent political party which has no connection or alliance with an English controlled party. (SNP, 1935, p. 3)

L'alinéa (4) s'adresse au Parti écossais qui ne fait preuve d'aucune forme d'exclusivité d'adhésion jusque-là. Cette résolution entre donc dans la lignée de la SNL puis du NPS, en plus d'ancrer la stratégie du SNP dans une dynamique de compétitivité électorale — par opposition à la création d'un groupe de pression parlementaire. Nous notons également que cet alinéa constitue l'expression de la volonté d'être représenté politiquement au parlement britannique, sans affiliation aucune à un parti à dominance anglaise. La définition du SNP comme parti expressément écossais, à la fois par son appellation et par sa stratégie politique, participe donc à l'affirmation identitaire du parti. Les trois alinéas précédents s'adressent aux anciens membres du NPS. En effet, ces derniers doivent adopter une position plus souple concernant l'auto-détermination écossaise afin d'attirer les anciens membres du SP. Selon l'alinéa (2), le SNP se voue à l'obtention d'une autonomie au sein de l'Empire britannique. Il est toutefois nécessaire de garder à l'esprit le caractère ambigu de la première résolution : si le parlement écossais doit constituer l'autorité finale sur l'ensemble des affaires écossaises, cela laisse apparaître en filigrane que la finalité politique du SNP n'est pas seulement l'obtention d'une forme d'autonomie limitée, mais que la conception d'une indépendance totale est envisagée. L'étendue des compromis entre NPS et SP 
reste néanmoins source de nombreuses confrontations entre les éléments radicaux et modérés.

Bien que le SNP témoigne d'une volonté de se distancier de la montée d'idéologies extrémistes telles que le fascisme et le nazisme à l'approche de la Seconde Guerre mondiale, la popularité du parti n’est pas favorisée par les stratégies d'action prônées par les éléments les plus radicaux du parti. La question de la conscription, voire de la participation à l'effort de guerre, est également problématique pour les nationalistes. Alors que certains défendent la position que le gouvernement britannique ne peut déclarer la guerre au nom des Écossais — bien qu'ils ne soient pas insensibles à la situation européenne — d'autres affirment qu'une opposition à l'effort de guerre contribue à établir une image antipatriotique et nuisible. En plus de valoir à Arthur Donaldson un emprisonnement de 40 jours en $1941^{22}$, la radicalisation du parti est complétée par l'élection ${ }^{23}$ de l'indépendantiste Douglas Young au poste de dirigeant du SNP en 1942. Las du sectarisme partisan et des faibles résultats électoraux, John MacGormick quitte le SNP, appelle les sympathisants de l'autonomie gouvernementale limitée à le rejoindre et destine le reste de sa carrière au groupe de pression parlementaire SNG. La Convention est notamment célèbre pour le lancement du National Covenant qui reçoit le soutien de deux millions de signataires et qui n'est pas sans rappeler l'appellation du Covenant de 1638. Le SNP, qui se voit dispensé de ses éléments modérés, reste dans l'ombre de la Convention jusqu'aux années 1960. La sécession de MacCormick permet néanmoins au SNP d'affirmer son idéologie indépendantiste; idéologie qui restera dès lors de mise au fil de l'histoire du parti jusqu'à nos jours.

\section{Réalignement du nationalisme politique écossais}

Contrairement au climat d'avant-guerre, la seconde moitié du vingtième siècle se montre plus clémente aux faveurs électorales du SNP. Parmi ces circonstances favorables, il est souvent fait mention du déclin de l'Empire britannique, du mécontentement envers le bipartisme en Grande-Bretagne dans les années 1970, et des promesses de politiques autonomistes faites par le parti conservateur puis par le parti travailliste (Civardi, 1998;

22. NLS, Acc 3721/2/30, Lettre du Comité de défense pour Donaldson, 5 juin 1941.

23. Young est partisan de l'indépendance et opposé à la conscription. Power, soutenu par l'autonomiste John MacCormick, défend une approche participative à la Seconde Guerre mondiale. Young remporte l'élection à 33 voix contre 29 (Brand, 1978, p. 241). La faible majorité acquise par Young témoigne indéniablement de l'étendue des tensions stratégiques et idéologiques qui sévissent dans les rangs du parti. 
Devine, 2006). D'autres privilégient la transformation interne du SNP d'un parti marginal à un tiers parti, notamment par l'intermédiaire d'une restructuration organisationnelle dans les années 1960 et grâce à l'établissement de politiques jugées crédibles par l'électorat écossais dans les années 1970, dont les campagnes «It's Scotland's Oil» et «Poor Britons or Rich Scots?» (Brand, 1978; Levy, 1989; Leruez, 2000; Lynch, 2002).

Cependant, l'importance du réalignement du nationalisme politique écossais dont profite le SNP est souvent diminuée. À la mort de John MacCormick en 1960, la Scottish Convention s'éteint progressivement pour laisser le devant de la scène au SNP. En effet, le travail des autonomistes dans la première moitié du vingtième siècle, redoublé des promesses électorales du parti conservateur et du parti travailliste au sujet de la mise en place d'une Assemblée écossaise, ont permis de faire entrer le SNP dans la lignée des partis politiques conventionnels, au meilleur sens du terme, comme en témoigne les sept sièges, puis onze, remportés aux élections législatives de février puis d'octobre 1974. Néanmoins, il semble difficile de nier l'influence retenue par la radicalisation du mouvement nationaliste, bien que nous gardions à l'esprit que ce phénomène ne saurait suffire à expliquer la montée du Scottish National Party. La radicalisation du mouvement nationaliste est alimentée par la formation d'organisations extrémistes et terroristes auxquelles Andrew Currie fait allusion dans une lettre qu'il envoie au secrétaire d'État à l'Écosse : «[...] there is a latent violence within Scotland, particularly about the fringes of the independence movement. At present, moderates are in control within the Scottish National Party, the Patriots, and the 1320 Club. The situation could change very fast $[\ldots]^{24}$.» Cette radicalisation du paysage nationaliste est bénéfique pour le SNP puisqu'elle renforce l'aspect conventionnel de ce parti politique au programme démocratique, par opposition à d'autres organisations qui adoptent des moyens d'actions plus extrêmes.

L'extrémisme politique nationaliste prend un nouvel élan en 1966 lors de la création du Workers' Party of Scotland (WPS) par six dissidents du parti communiste britannique. Le WPS est notable par l'idéologie marxisteléniniste qu'il allie au républicanisme et au nationalisme dans les articles publiés au sein de son organe de communication Scottish Vanguard ${ }^{25}$. Alors

24. NLS, Acc 12509/3, Lettre d'Andrew Currie à Gordon Campbell, 9 décembre 1972. D'autres lettres basées sur le même modèle furent modifiées en fonction des destinataires et furent envoyées le même jour aux personnes suivantes : William Ross, Hamish Gray, Russell Johnston, David Steel et Billy Wolfe. Des copies de ces lettres furent transmises à Wendy Wood et aux Sottish Patriots, au SNP et au $1320 \mathrm{Club}$.

25. NLS, Acc 7295/29/5, Scottish Vanguard, 6 février 1968. 
que le WPS relève à première vue du radicalisme révolutionnaire ${ }^{26}$, le répertoire d'action dont il fait usage fait apparaître la teneur extrémiste du parti. Puisque la préparation de la révolution prolétarienne internationale que le WPS entend guider nécessite un apport financier considérable, la figure de proue du parti, Matthew Lygatte, accompagné de quelques associés, décident de braquer quatre banques de Glasgow et de sa périphérie, un réseau de transport et un fourgon appartenant au réseau ferroviaire britannique. Ces braquages (de mai à décembre 1971) réunissent 27000 livres sterling et sont perpétrés avec une violence extrême à l'aide de fusils à pompe, de pistolets et de couteaux, sans toutefois faire de blessés graves. Les cinq accusés sont condamnés par la Haute Court de Justice de Glasgow à une peine d'emprisonnement représentant un total de 84 ans (Macleay et Scott, 1990, p. 41-42).

Le radicalisme nationaliste continue son essor en 1967 avec la constitution du 1320 Club dont l'appellation fait référence à la déclaration d'Arbroath ${ }^{27}$. Tandis que la direction révolutionnaire et républicaine s'affirme sous la direction de Hugh MacDiarmid, Radio Free Scotland (RFS), créée par un petit nombre d'individus appartenant au SNP, déclare que le mouvement nationaliste est en guerre sous prétexte que le SNP n'est pas représenté dans les médias. Armée seulement d'un transmetteur courte portée installé dans un appartement de Glasgow, elle entend redresser la situation par la création d'une radio pirate dont l'objet est de disséminer la propagande du SNP (Radio Free Scotland, n. d., p. 1). Peu après, Wendy Wood, dont le parcours nationaliste radical a été marqué par son action au château de Stirling dans les années 1930 (elle décroche l'Union Fack et le remplace par le Lion Rampant), décide en 1972, à l'âge de 82 ans, d'entamer une grève de la faim en vue d'obtenir une concrétisation de la promesse électorale ${ }^{28}$ faite par Edward Heath lors de la déclaration de Perth. Tandis que Wendy Wood séduit une partie des Écossais qui lui envoient des lettres de soutien, d'autres adressent au Premier ministre une série d'avertissements. Le moyen d'action dont use Wendy Wood, qui

26. «On évoque souvent le radicalisme révolutionnaire pour désigner, durant les périodes de renversement du pouvoir, les éléments les plus intransigeants, les plus extrémistes, qui souhaitent une rupture totale avec le passé, c'est-à-dire un changement radical de société qui suppose, le plus souvent, l'abandon de l'ensemble des institutions, des règles et des traditions héritées de l'histoire.» (Nay, 2008, p. 460)

27. «For as long as but a hundred of us remain alive, never will we on any conditions be brought under English rule. It is not for glory, nor riches, nor honours that we are fighting, but for freedomfor that alone, which no honest man gives up but with life itself.»

28. Voir <www.scotsman.com/news/four-decades-on-declaration-of-perth-is-still-fuelling-debate1-1169195>. Heath déclare : «... a Constitutional Committee should be set up to examine proposals for the re-organization of Scottish Government. [...] We would propose to the Constitutional Committee the creation of an elected Scottish Assembly, to sit in Scotland.» 
est plus extrême qu'il n'est radical, sert donc de moteur aux éléments les plus en marge du mouvement nationaliste. Preuve en est de cet extrait de la lettre envoyée par le président honoraire du 1320 Club et dont de brefs passages sont relayés dans le journal The Guardian. Initialement écrite en anglais, des traductions de l'intégralité de la lettre «seront adressées aux journaux européens les plus importants ${ }^{29}{ } »$ :

Nous n'ignorons pas qu'il existe dans ce pays (et par «ce pays» je veux dire l'Écosse) des organisations «activistes» demi-secrètes qui sont tout à fait prêtes à créer une situation violente si vous continuez encore longtemps vos tactiques d'attente. Les Écossais sont un peuple dont les réflexes sont impossibles à prévoir — un peuple que vous les anglais [sic] ne pouvez aucunement comprendre; et vous seriez bien avisé de tenir compte de l'agitation souterraine. L'Écosse en a assez des affronts, des promesses manquées, et des mensonges éclatants des Anglais. Soyez donc prévenus de la situation potentiellement explosive avec laquelle vous avez affaire ${ }^{30}$.

La situation devient réellement explosive lorsque, en soutien à la contestation dont fait preuve Wendy Wood, le Border Clan revendique la destruction par explosif d'un pied d'un pylône électrique le 9 décembre 1972. Le gouvernement conservateur, qui n'a pas l'intention de faire de la chef des Scottish Patriots une martyre, annonce la publication d'un livre vert et accède à la revendication de Wendy Wood, alors dans son septième jour de grève. Il est discutable d'attribuer le résultat à l'organisation terroriste $^{31}$ Border Clan en raison de la lutte anti-terroriste intense déjà portée par le gouvernement.

Le SNP tient à garder ses distances avec la radicalisation du mouvement nationaliste et expulse de ses rangs le major Boothby en raison de la teneur gênante des propos radicaux qu'il soumet, à savoir l'établissement d'un «Cabinet fantôme» en Écosse ${ }^{32}$. Boothby s'engage dans la voie du terrorisme lorsqu'il prend la tête de l'Army of the Provisional Government (APG) dont le répertoire d'action est le suivant : «[d]rugs would be banned, the Gaelic culture reinforced, all government buildings levelled, the Common Market abandoned and certain "undesirables" deported" (Hewitson, 1975, p. 5). Il est intéressant de rappeler que Boothby mentionne une certaine Scottish Liberation Army (SLA) dans le premier numéro

29. NLS, Acc 12509/3, Communiqué de presse signé par R. MacDonald Douglas, 4 décembre 1970 .

30. Ibid.

31. Le terrorisme se traduit dans les «[a]ctivités de groupes clandestins et organisés faisant le choix d'actions particulièrement violentes (assassinats, attentats, séquestrations, enlèvements, prises d'otage, sabotages, etc.) contre des intérêts politiques, économiques ou religieux» (Nay, 2008, p. 552).

32. NLS, Acc 10090/106, Lettre de Frederick A. C. Boothby à Robert McIntyre, 4 avril 1963. 
du journal grand format Skian Dhu qu'il édite dès $1963^{33}$. En effet, le major (appellation qu'il préfère à son nom de famille) publie des nouvelles d'une armée mystérieuse qui n'hésite pas à faire appel à des dons de matériel (du tapis de sol au télescope en passant par les vêtements, gris ou kaki de préférence) et à proposer, sous forme d'articles, des conseils pour préparer une lutte armée ou bien pour éviter les chiens pisteurs ${ }^{34}$. La SLA disparaît soudainement après la parution du neuvième numéro. Tandis que l'existence de la SLA dans les faits reste discutable, la formation de l'APG dès 1971 ne fait aucun doute.

À elle seule, l'analyse des propos tenus au cours des années 1970 par les acteurs de la scène politique présente le major Boothby sous des angles différents qui ne sauraient convenir à une classification objective. En effet, tandis que les membres de l'APG considèrent qu'ils œuvrent dans le cadre d'une guerre de libération face au joug que retient l'Angleterre sur l'Écosse (Hewitson, 1975, p. 4), la police britannique mène plusieurs arrestations sous les auspices du Prevention of Terrorism Act, 1974 avant que les suspects, dès lors étiquetés «extrémistes ${ }^{35}$ », ne soient jugés pour conspiration, possession d'arme à feu et d'explosifs avec intention de nuire et qu'ils ne soient emprisonnés. Les cadres dirigeants du SNP n'hésitent pas à qualifier le major dans les termes suivants : «the man is either mentally off balance and therefore not normally honest or he is a double agent ${ }^{36} »$. Le président du 1320 Club, Ronald Douglas MacDonald, se contente de voir en Boothby une «menace ${ }^{37} »$, «a ruthless megalomaniac, a double dealer and a blackmailer ${ }^{38}$ » si ce n'est un agent provocateur envoyé par les services secrets britanniques afin de noircir l’image du nationalisme écossais ${ }^{39}$. Alors que le ministère de la santé en

33. Acc 10090/107, Skian Dhu, vol. 1, n 1, F. A. C. Boothby, septembre 1963, p. 3. Le journal s'intitule Skian Dhu au cours des neuf premiers numéros avant de retrouver la graphie Sgian Dubh, plus courante mais sans changement de prononciation. Boothby explique que ce choix est délibéré et qu'il vise à initier ses lecteurs à la prononciation du gaélique.

34. Acc 10090/107, Skian Dhu, vol. 1, n 4, F. A. C. Boothby, janvier 1964, p. 8. Acc 10090/107, Skian Dhu, vol. 1, no 7, F. A. C. Boothby, avril 1964, p. 5.

35. NAS, HH16/548, Report from the Scottish Home and Health Department, 8 avril 1976 : «The trial at which he [Boothby] and others were sentenced attracted considerable publicity and demonstrated the existence, in Scottish affairs, of extremists who were apparently prepared to adopt very violent methods in the furtherance of political aims. There is no doubt that Boothby was involved in at least some of the activities."

36. Acc 10090/106, Lettre d'Arthur Donaldson à Robert McIntyre, 3 novembre 1965. Une copie est envoyée à Gordon Wilson.

37. Acc 8509/3, Lettre de Ronald Douglas MacDonald à John Herdman, 5 octobre 1971.

38. Acc 8509/3, Lettre de Ronald Douglas MacDonald à John Herdman, 10 octobre 1971.

39. Ibid. : «Furthermore, he must be forced out of Scottish life. He is, to begin with, not a Scotsman; and I fully believe that he was sent to this country to disrupt the SNP, and when the SNP flung him out, he was subsidised by his masters to put out his so-called Newsletter. He then joined the Scottish 
charge de rédiger le rapport relatif à l'équilibre mental du major le considère comme «a rather ineffective political enthusiast who was used by criminal elements in the APG rather than a director of their activities ${ }^{40}$ », la branche spéciale de la police de Strathclyde, à l'inverse, stipule :

Although the subject is 65 years of age, he was the figurehead of the Army of the Provisional Government and there is little likelihood that he has altered his extreme Nationalistic views. The open conditions may allow him to renew associations with extremist colleagues while unsupervised ${ }^{41}$.

Il revient donc au chercheur de trancher sur l'appellation à attribuer au major et à l'APG. Le procès des hommes impliqués dans l'APG, qui se déroule en mai 1975, permet de poursuivre notre réflexion sur la base de faits objectifs. Bien que le procès-verbal et les pièces à conviction soient scellés sous le Data Protection Act jusqu'en 2045, le dossier de demande de remise en liberté sur parole du major Boothby, lorsqu'il est mis en relation avec les articles de presse parus au cours du procès, est révélateur de l'orientation adoptée par les figures emblématiques de l'APG, qui présentent des connexions avec les organisations terroristes parallèles. William Anderson, dont s'inspire James Robertson pour l'«ex-REME soldier» (Royal Electrical \& Mechanical Engineer - REME) mentionné dans l'extrait cité en début d'article, avait appris à manipuler les explosifs avant de s'associer au major. William Murray a poursuivi son implication dans l'APG, y compris après avoir purgé une première peine d'emprisonnement durant laquelle il fit la connaissance de Matthew Lygatte du WPS et de Tony Tunilla. La sœur de ce dernier entretenait une relation sentimentale avec le dirigeant terroriste de l'Army of the Scottish People, Peter Wardlaw, qui préconisait les assassinats et les attentats (Thomson et Alden, 1975, p. 9; Macleay et Scott, 1990, p. 83-105). Par ailleurs, l'APG tenta une campagne médiatique en 1974 lorsqu'elle invita trois journalistes du Daily Record à un sommet comprenant les représentants des associations terroristes écossaises, à savoir la SLA, la Scottish Citizen Army of the Republic (SCAR) et la Scottish Republican Army (SRA). Toutefois, les actions de l'APG, dont deux tentatives de braquage de banque en 1971 et 1975, se soldèrent par des échecs en raison de la présence d'informateurs et d'agents provocateurs dans les rangs de l'organisation terroriste (Macleay et Scott, 1990, p. 52-53). S'il n'a pas été prouvé que

\footnotetext{
Plebiscite Society and, according to the information I can get, he was responsible for its destruction. He has destroyed other things too.»

40. NAS, HH16/548, Report from the Scottish Home and Health Department, 8 avril 1976.

41. NAS, HH16/548, Lettre de la Branche spéciale de la police de Strathclyde au directeur de prison, 20 février 1976.
} 
Boothby a agit en tant que dirigeant de l'organisation, le major en reste néanmoins l'une des figures médiatiques. Eu égard des éléments factuels avancés et en accord avec les définitions linguistiques des concepts servant à établir le cadre conceptuel de notre typologie, il transparaît que l'extrémisme de Boothby en tant qu'éditeur de Skian Dhu bascule dans le terrorisme dès que le major rejoint l'APG. En effet, même si l'établissement d'un Cabinet fantôme en Écosse témoigne d'une volonté d'agir de manière clandestine, il s'agit d'abord, pour reprendre la définition que nous avons adoptée, d'une forme d'action directe visant à dénoncer le fonctionnement de la démocratie représentative. À l'inverse, les relations qu'entretiennent les membres de l'APG, ainsi que le répertoire d'action de l'organisation, s'inscrivent dans une dynamique terroriste qui ambitionne le renversement des intérêts politiques britanniques et l'établissement d'un nouveau mode de gouvernement de l'Écosse (Thomson et Alden, 1975, p. 9).

Nous gardons à l'esprit que le SNP n'a pas été exempt d'un factionnalisme intensif à la suite du référendum de 1979 et que les divisions idéologiques ont mis à mal la cohésion du parti. Tandis que Campaign for Nationalism in Scotland (CNS) se forme pour contrecarrer l'idéologie socialiste et républicaine de la faction radicale 79 Group qui utilise la désobéissance civile comme moyen d'action (Ascherson, 1979, p. 7), la faction Siol Nàn Gàidheal (SNG) affiche une forme d'extrémisme politique lorsqu'elle n'hésite pas à s'écarter des lignes de conduite dictées par le SNP, tout en défiant l'autorité policière, lors de ses défilés militaristes. Qualifiée de «guerre ouverte» (Baur, 1982, p. 1), la descente du parti dans le factionnalisme interne voit la CNS qualifier le 79 Group de parasite, tandis que Gordon Wilson, alors dirigeant du parti, confie ultérieurement qu'il jugeait que les membres du SNG étaient des «protofascistes» (Wilson, 2009, p. 205). Il faudra alors attendre l'exclusion des factions en 1982 pour que le parti puisse retrouver une cohérence idéologique et stratégique afin de poursuivre une ligne politique dont le conventionnalisme est favorisé par la création de la Scottish Republican Socialist League (SRSL) en 1979, puis du Scottish Republican Socialist Party (SRSP) en 1987. En effet, ces deux organisations entretiennent le radicalisme révolutionnaire du mouvement républicain avancé dix ans plus tôt par le 1320 Club, l'une œuvrant en vue de rendre le pouvoir à la classe ouvrière qui mettrait fin aux intérêts impérialistes des Anglais et des Américains, l'autre prônant une stratégie de retrait du parlement de Westminster et la création d'une assemblée écossaise (Scottish Martyrs Committee, 1980, p. 7 ; SRSP, 1987, p. 1). 
S'il n'a pas été possible de traiter les manifestations nationalistes dans leur intégralité et que le rôle primordial joué par la dimension culturelle du nationalisme écossais n'a pas été abordé, cela ne signifie en aucun cas que leur influence doive être diminuée. L'angle d'étude que nous avons privilégié permet néanmoins d'enrichir la délimitation des idéologies nationalistes par une classification onomastique des formations politiques en vue de présenter le mouvement nationaliste dans son unité et sa fragmentation.

Placée sous l'angle de l'échiquier politique britannique, toute revendication nationaliste formulée au sein du cadre institutionnel existant et sans violence est radicale. En effet, elle se manifeste par une attitude réformiste visant un changement profond du système gouvernemental en Écosse. À l'inverse, l'extrémisme politique se développe à la limite de ce cadre institutionnel et reste susceptible de basculer dans le terrorisme. Ce dernier éclot en raison de l'usage de méthodes invariablement violentes et hors de tout cadre institutionnel et législatif.

Il apparaît que le nationalisme politique écossais dans la première moitié du vingtième siècle relève du radicalisme et de l'extrémisme. Tandis que le SNP peine à s'affirmer idéologiquement et stratégiquement dès sa création, le parti reste une formation politique radicale qui est parvenue à faire entrer ses revendications indépendantistes dans le conventionnalisme du jeu politique britannique, notamment à partir des années 1960 et en raison de sa modération politique en regard de la radicalisation du mouvement nationaliste apportée par la formation d'organisations terroristes et extrémistes dans les années 1970 et 1980 .

\section{Bibliographie}

\section{Articles de journaux}

Ascherson Neal, «SNP Faction Affirms Policy», The Scotsman, 20 août 1979 , p. 7.

Baur Chris, «Open War Breaks Out in SNP», The Scotsman, 4 juin 1982, p. 1.

Hewitson Jim, «The APG Policy», Glasgow Herald, 24 mai 1975, p. 4. Thomson Drew et Alden Jane, «The Blueprint for Terror», Scottish Daily Mirror, 6 mai 1975, p. 9.

\section{Fuvre littéraire}

Robertson James, And the Land Lay Still, Londres, Hamish Hamilton Limited, 2010. 


\section{Sources primaires}

Archives nationales d'Écosse :

HH16/548, Criminal Case File: Major Frederick Alexander Colquhoun Boothby ('Tartan Army' Case), 1975-1976.

Laughland William, Manifesto and Appeal to the Scottish People on Scottish Home Rule, Glasgow, Young Scots Society, juillet 1911.

SNP, Constitution and Rules 1934-1935, Glasgow, Wm McLellan \& Co Ltd, 1935.

Spence Lewis, Freedom for Scotland: The Case for Scottish Self-Government, Édimbourg, SNM, 1927.

Radio Free Scotland, End the conspiracy of silence, Glasgow, Radio Free Scotland, n. d.

The Scottish Martyrs Committee, Commemoration March for Fames Wilson and the Other Martyrs of the 1820 Insurrection, Édimbourg, SRSL, 17 mai 1980.

SRSP, Declaration of Aims and Principles and Articles of Association, Glasgow, SRSP, 1987.

Bibliothèque nationale d'Écosse :

Acc 3721, Papers of the Scottish Secretariat and of Roland Eugene Muirhead. Acc 6058, Correspondence and Papers of T. H. Gibson.

Acc 7295, Scottish National Party.

Acc 8509, Fohn Herdman.

Acc 10090, Papers of Dr Robert Douglas McIntyre.

Acc 12509, McIntyre (Robert Douglas), President, Scottish National Party.

Dep 209, Neil M. Gunn LL.D.

\section{Sources secondaires}

BRAnd Jack, The National Movement in Scotland, Londres, Routledge \& Kegan Paul, 1978.

Civardi Christian, L'Écosse depuis 1528, Gap, Orphys, 1998.

Coleman Janet, Connoly William, Miller David et Ryan Alan (éds), Dictionnaire de la pensée politique. Hommes et idées, Paris, Hatier, 1989.

Devine Thomas Martin, The Scottish Nation 1700-2007, Londres, Allen Lane, 2006.

DupRIEz Bernard, Gradus : les procédés littéraires (dictionnaire), Paris, Union générale d'éditions, 1984.

FInLAY Richard, Independent and Free, Édimbourg, John Donald, 1994.

Himelfarb Georges, Sigles et Acronymes, Paris, Belin, 2002.

Kennedy James, Liberal Nationalisms: Empire, State, and Civil Society in Scotland and Quebec, Montreal, McGuill-Queen's University Press, 2013. 
Leruez Jacques, L'Écosse. Vieille nation, jeune État, Crozon, Armeline, 2000.

Levy Roger, Scottish Nationalism at the Crossroads, Édimbourg, Scottish Academic Press.

Lynch Peter, SNP: The History of the Scottish National Party, Cardiff, Welsh Academic Press, 2002.

Macleay Ian et Scott Andrew Murray, Britain's Secret War: Tartan Terrorism and the Anglo-American State, Édimbourg, Mainstream Publishing Company Ltd, 1990.

Mitchell James, The Scottish Question, Oxford, Oxford University Press, 2014.

NAY Oliver (Dir.), Lexique de Science politique. Vie et institutions politiques, Paris, Dalloz, 2008.

Thomsen Robert G., Nationalism in Stateless Nations, Édimbourg, Birlinn Ltd, 2010.

WiLson Gordon, SNP: The Turbulent Years 1960-1990, Stirling, Scots Independent (Newspapers) Ltd, 2009.

\section{Liste des sigles et acronymes}

APG : Army for the Provisional Government

BBC : British Broadcasting Corporation

CNS : Campaign for Scottish Nationalism

GUSNA : Glasgow University Student Nationalist Association

ILP : Independent Labour Party

NPS : National Party of Scotland

REME : Royal Electrical \& Mechanical Engineer

RFS : Radio Free Scotland

SCAR : Scottish Citizen Army of the Republic

SHRA : Scottish Home Rule Association

SI : Scots Independent

SLA : Scottish Liberation Army

SNC : Scottish National Convention

SNL : Scottish National League

SNM : Scottish National Movement

SNP : Scottish National Party

SP : Scottish Party

SRA : Scottish Republican Army

SRSL : Scottish Republican Socialist League

SRSP : Scottish Republican Socialist Party

SNG : Siol Nàn Gàidheal

WPS : Workers' Party of Scotland 